\title{
Fluid motion and solute distribution around sinking aggregates. I. Small-scale fluxes and heterogeneity of nutrients in the pelagic environment
}

\author{
Thomas Kiørboe $^{1, *}$, Helle Ploug ${ }^{2}$, Uffe H. Thygesen ${ }^{1}$ \\ ${ }^{1}$ Danish Institute for Fisheries Research, Kavalergården 6, 2920 Charlottenlund, Denmark \\ ${ }^{2}$ Marine Biological Laboratory, Strandpromenaden 5, 3000 Helsingør, Denmark
}

\begin{abstract}
Marine snow aggregates are sites of elevated biological activity. This activity depends on the exchange of solutes $\left(\mathrm{O}_{2}, \mathrm{CO}_{2}\right.$, mineral nutrients, dissolved organic material, etc.) between the aggregate and the environment and causes heterogeneity in the distribution of dissolved substances in the ambient water. We described the fluid flow and solute distribution around a sinking aggregate by solving the Navier-Stokes' equations and the advection-diffusion equations numerically. The model is valid for Reynolds numbers characteristic of marine snow, up to $R e=20$. The model demonstrates the importance of a correct flow environment when making biological rate-measurements on aggregates (e.g., oxygen consumption/production, growth rates of bacteria and phytoplankton) because both solute fluxes and internal solute concentrations depend strongly on the flow environment. Observations of flow and oxygen-concentration fields in the vicinity of both artificial and natural oxygen-consuming aggregates that are suspended in a flow compare well with model predictions, thus suggesting that our set-up is suitable for making biological rate measurements. The sinking aggregate leaves a long slender plume in its wake, where solute concentration is either elevated (leaking substances) or depressed (consumed substances) relative to ambient concentration. Such plumes may impact the nutrition of osmotrophs. For example, based on published solubilization rates of aggregates we describe the amino acid plume behind a sinking aggregate $(0.1$ to $1.0 \mathrm{~cm}$ radius). The volume of the plume with amino acid concentrations high enough to significantly affect bacterial uptake rates is ca $100 \times$ the volume of the aggregate itself. Thus, sinking aggregates may create significant microniches also for free-living bacteria.
\end{abstract}

KEY WORDS: Marine snow · Mass transport - Bulk Sherwood number · Local Sherwood number · Aggregate respiration rate

Resale or republication not permitted without written consent of the publisher

\section{INTRODUCTION}

Aggregation of suspended particles into large rapidly sinking aggregates is believed to be one of the main processes driving vertical material fluxes in the ocean (Fowler \& Knauer 1986). Aggregates are also sites of enhanced biological activity. Within the euphotic zone aggregated phytoplankton may be photosynthetically active (Knauer et al. 1982). Throughout the water col-

*E-mail: tk@dfu.min.dk umn bacteria hydrolyse (Smith et al. 1992, Grossart \& Simon 1998) and respire (Ploug et al. 1999a) aggregated particulate matter at high rates, protozoan communities develop and feed on bacteria and other small particles inside aggregates (Caron et al. 1982, Turley \& Mackie 1994, Ploug \& Grossart 2000), and zooplankton colonize and inhabit aggregates while feeding on their constituents (Alldredge 1976, Shanks \& Walters 1997, Kiørboe 2000). A substantial fraction of aggregated material may therefore be remineralized before the aggregate leaves the upper mixed layer (Kiørboe 2000). The degradation processes continue below the 
mixed layer and contribute to the observed decline in particle flux with depth (Martin et al. 1987, Pace et al. 1987). Hence, the formation and degradation of sinking aggregates have major implications for both the retention and export of limiting elements in the upper ocean, and for the ocean's carbon and nutrient budgets.

The biological activities in sinking aggregates are governed by the exchange of solutes (e.g., $\mathrm{CO}_{2}, \mathrm{O}_{2}$, inorganic nutrients, and hydrolysis products) between the aggregate and the ambient water through flow and molecular diffusion in the vicinity of the aggregate. The growth of aggregated phytoplankton may depend on the transport of inorganic nutrients towards the aggregate; aggregate remineralization rate can be limited by the transport rate of oxygen towards the aggregate; internal concentrations of solutes such as oxygen and dissolved organics, which are produced and/or consumed inside aggregates, depend on the rate of transport to/from the aggregate and, hence, govern the rate of concentration-dependent processes. Finally, aggregates leaking remineralization and hydrolyzation products (DOM, inorganic nutrients) may leave a trail of enhanced solute concentration during sedimentation. Such trails may provide a cue to zooplankters that search for aggregates, and may generate heterogeneity in the ambient distribution of solutes utilised by phytoplankton and free-living bacteria. To understand the degradation processes listed above, one must therefore know the advective and diffusive exchange of solutes between the aggregate and the ambient water. Furthermore, interpretation of experimental measurements depends on a knowledge of the effects and time scales of transport processes.

Flow and solute distributions around sinking particles and, hence, mass transport have been described in the oceanographic literature (Csanady 1986, Jackson 1989, Karp-Boss et al. 1996, and references therein) but have assumed Stokes' flow and are hence valid only for systems at low Reynolds numbers $(R e \ll 1)$, i.e., small and slow-sinking particles. Marine snow aggregates are typically larger, faster sinking and characterised by $R e$ up to about 20 (Alldredge \& Gotschalk 1988). Here we develop from first principles a numerical model of the flow and solute distribution around sinking particles valid up to $R e=20$, thus encompassing the typical range of marine snow particles. We compare model predictions with direct observations of flow and solute distributions around aggregates suspended in flow. Hereby we evaluate the experimental system, which previously has been used to estimate oxygen consumption and production in aggregates (Ploug \& Jørgensen 1999). The results are discussed in relation to small-scale fluxes of solutes between sinking aggregates and the ambient water during sedimentation and the heterogeneity of organic matter and solutes in the ocean.

\section{MATERIAL AND METHODS}

The model. The sinking marine snow aggregate is modelled as a solid sphere moving through the water at constant speed. The flow is described by solving the Navier-Stokes' equations numerically, and the solute distribution is described by then solving the governing diffusion-advection equation. Diffusion and advection govern the concentration field and the resulting solute exchange in an identical manner, whether the aggregate is a sink or a source of a particular solute. A more detailed description of the model and of the numerical approach can be found in Appendix 1. Here we provide only a few necessary definitions.

Following the convention of Karp-Boss et al. (1996) we take the radius of the sphere $a$ as the characteriststic length, and the free flow $U$ as the characteristic velocity. The flow and the concentration fields are then described by the Reynolds number

$$
R e=\frac{a U}{v}
$$

and the Peclet number

$$
P e=\frac{U a}{D}
$$

where $v$ is the kinematic viscosity and $D$ the diffusion coefficient. We use SI units for the quantities $a_{1} U, v$ and $D$, although any consistent system of units can be used. The dimensionless numbers $R e$ and $P e$ are related by the Schmidt number,

$$
S_{C}=\frac{P e}{R e}=\frac{v}{D}
$$

To calculate the solute distribution, we investigated 2 alternative inner boundary conditions, i.e., either that the concentration is constant over the surface of the sphere (Dirichlet boundary condition) or that the flux is constant over the surface of the sphere (Neumann boundary condition). Traditionally, a Dirichlet condition has been assumed in oceanographic work (KarpBoss et al. 1996). The Dirichlet condition corresponds to the case where solute transfer and, hence, the rate of the relevant biological process is transport limited and the flux therefore increases with increasing fluid flow. The Neumann condition corresponds to the situation where the flux is limited by the rate of the biological process (reaction limitation) and the concentration at the surface depends on the fluid flow. Comparison of the observations with predictions based on these alternative boundary conditions thus offers a 
way of assessing whether solute flow is reaction or transport limited.

Experiments. Near spherical, oxygen-consuming model aggregates, 3 to $4 \mathrm{~mm}$ in diameter, were made as described by Cronenberg, 1994: Bakers yeast was suspended in a $2 \%(\mathrm{w} / \mathrm{w})$ agar solution at $+40^{\circ} \mathrm{C}$ and dripped by a Pasteur pipette into thin paraffin oil at room temperature. An almost perfect agar-yeast sphere forms from each drop. The spheres were rinsed and stored for up to a few days in freshwater. More typical marine snow aggregates were produced in a rolling tank using detritus collected from a copepod culture (Acartia tonsa grown on Rhodomonas baltica). We selected for measurements the 'most' spherical aggregates, which were, however, often somewhat oblate and with an irregular surface. All measurements with detritus aggregates were made in seawater, while measurements with yeast aggregates were made in freshwater. Experimental temperature varied between experiments, ranging from 7 to $20^{\circ} \mathrm{C}$, corresponding to $S_{C}$ between ca 450 and 1000. Additional measurements on detritus aggregates and field-sampled marine snow were taken from Ploug (unpubl.) and Ploug et al. (1999a).

Flow and solute distributions were measured around aggregates suspended in an upward-directed flow generated in the flow-through chamber described by Ploug \& Jørgensen (1999). Briefly, the chamber consisted of a $10 \mathrm{~cm}$ long cylindrical Plexiglas tube, inner diameter (i.d.) $5 \mathrm{~cm}$, with a nylon stocking (100 to $300 \mu \mathrm{m}$ mesh size) distended across the middle of the tube. Water was supplied from below, passed the mesh, and left the cylinder through 4 opposing outlets at the top of the chamber. The entire chamber was submerged in a constant temperature water bath. The aggregate was mounted on a very thin $(30 \mu \mathrm{m})$ glass thread and suspended about $5 \mathrm{~mm}$ above the mesh. Additional measurements were conducted in a scaledup version of the chamber $(10 \mathrm{~cm}$ i.d., $10 \mathrm{~cm}$ long above the net) with aggregates suspended further away from the mesh (2 to $4 \mathrm{~cm}$ ).

To visualise the flow, the aggregate was illuminated from the side by a horizontally oriented 0.6 to $0.7 \mathrm{~mm}$ thick Ne-laser sheet, and the movements of neutrally buoyant particles (27 $\mathrm{mm}$ Locopodium pollen) were recorded by a video camera oriented perpendicular to the laser sheet. The camera was equipped with a $105 \mathrm{~mm}$ macrolens and connected to a VCR with a video timer $(1 / 100 \mathrm{~s})$. Thus, flow in one azimuthal plane was observed, but is assumed representative of the near 3-dimensional radially symmetric flow. Particle positions were digitised to obtain streamlines and flow velocity distributions. Flow velocities were computed as 0.1 to $0.3 \mathrm{~s}$ averages (depending on their magnitude). We concentrated on flow velocities on tran- sects off the aggregate's equator and directly up- and downstream of the aggregates. Due to the thickness of the laser sheet, apparent distances of particles from the aggregate are overestimated on average. Because the flow is radially symmetric, apparent particle distances directly off the aggregate's equator could be converted to average real distance (see Kiørboe \& Titelman 1998); a similar conversion was not possible in other directions and, hence, distance-specific velocities are slightly overestimated here on average, particularly close to the aggregate.

Oxygen gradients were measured at steady-state in transects off the equator (i.e., perpendicular to the flow direction) and directly downstream of the aggregate by means of a long, slender Clark-type microelectrode with a $4 \mu \mathrm{m}$ sensing tip, a $90 \%$ response time $<0.5 \mathrm{~s}$, and a stirring sensitivity of $0.3 \%$ (Revsbech 1989). The microelectrode was fixed on a micromanipulator and the position of the aggregate surface was determined by slowly moving the tip of the electrode towards the aggregate until it touched the surface as observed on the video image (ca $25 \times$ magnification). Measurements along the transects were taken at 50 to $1000 \mu \mathrm{m}$ intervals (depending on the gradient) from the surface until no further change in oxygen concentration could be measured. The oxygen fluxes and local Sherwood numbers were calculated from the measured gradients at the aggregate-water interface as described earlier (Ploug et al. 1997).

\section{RESULTS AND DISCUSSION}

\section{Flow field}

Stokes' flow is perfectly symmetric (Fig. 1). With increasing $R e$, the computed flow becomes increasingly asymmetric: velocity gradients become steeper in front of and particularly along the sides of the sphere, while the flow velocity is reduced downstream of the particle (see also Fig. 2). Vorticity isolines are advected downstream. Recirculation downstream becomes evident at $R e \approx 12$ (not shown). Streamlines and spatial distribution of vorticity at various $R e$ replicate accurately those computed by Dennis \& Walker (1971) and Chang \& Maxey (1994), and the onset of recirculation (separation) at $R e \approx 12$ is consistent with both experimental observations and models (Johnson \& Patel 1999). Thus, we are confident that our numerical results are valid.

The flow velocity profiles recorded in our experimental system are in reasonable accord with the model (Fig 2): the flow becomes increasingly asymmetric with increasing $R e$ and the velocity gradients increase upstream and off the sphere's equator and decrease 

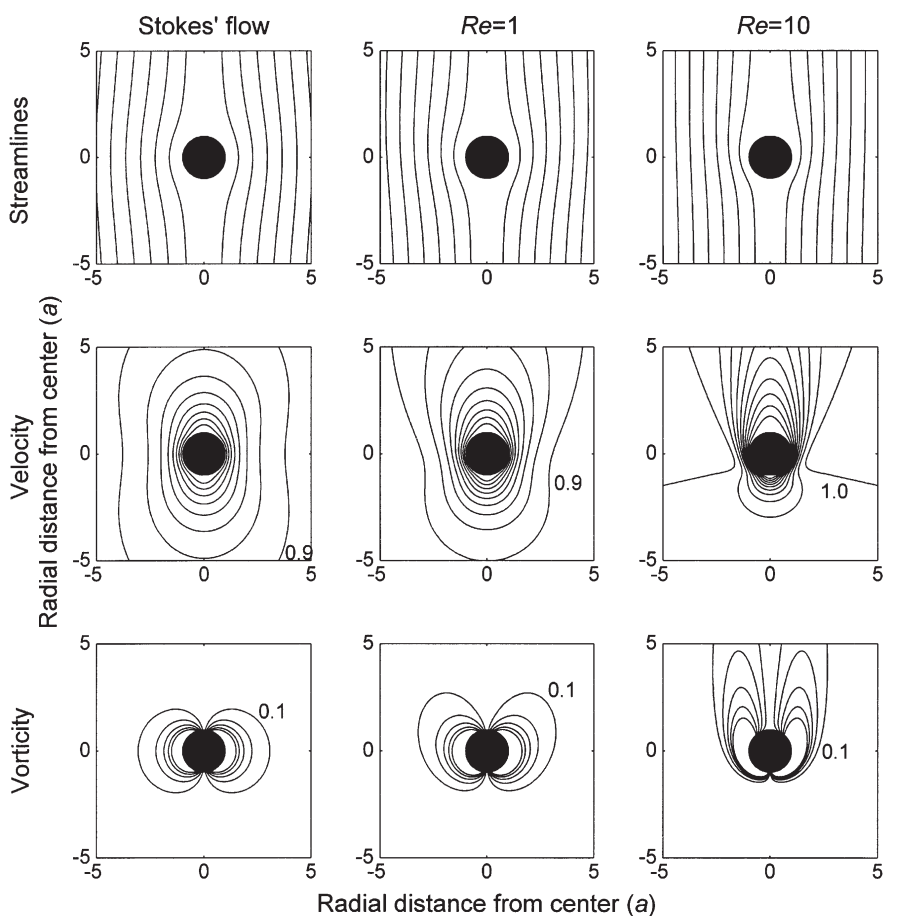

Fig. 1. Streamlines and distribution of velocity and vorticity around sinking sphere for Stokes' flow and at $R e=1$ and 10. The particle is moving down. Distances are in units of radii. Velocity isolines (units of $U$ ) and vorticity isolines (units of $U / a$ ) are in steps of 0.1 (the outermost isolines are labelled)

downstream. The model predicts a slight peak in flow velocity off the sphere's equator where the flow velocity slightly exceeds the flow velocity far away (evident in Fig. 2H). This peak is sometimes also seen in the data, but the observed peak is at times larger than that predicted (e.g., Fig. 2F), leading to slightly steeper velocity gradients off the equator. Also, some distance downstream, the observed flow exceeds the predicted flow. This is because the flow lines in the chamber were not perfectly parallel due to unavoidable slight instabilities in the flow. This also caused the undisturbed flow velocities in our chamber to vary by up to $20 \%$. Thus, the flow around the particle in our experimental system shares essential features with the flow around a freely falling particle, but does not replicate it exactly.

\section{Concentration field}

We can describe the spatial distribution of solutes consumed by (or leaking from) aggregates by means of the model (Fig. 3, here computed for $S C=1000$ ). At $P e=0$ (i.e., a non-sinking sphere) solute transfer is governed by diffusion only, and the resulting solute distribution is radially symmetric. With increasing $P e_{\text {, }}$ the concentration distribution becomes increasingly asymmetric with a long slender plume of reduced concentration at the rear of the aggregate. By $P e=100$ $(R e=0.1)$ the plume is very long and slender and 20 radii downstream the concentration is still reduced by more than $10 \%$ of the reduction at the surface. Higher $P e$ yields even longer plumes. We are unaware of previous attempts to model solute distributions at $R e>0.1$ and $S C$ relevant to biological oceanography $(\sim 400$ to 4000 for sugars, amino acids, oxygen, nutrient salts between 0 and $25^{\circ} \mathrm{C}$ ).

The observed oxygen concentrations measured off the equator of our yeast aggregates agree with those
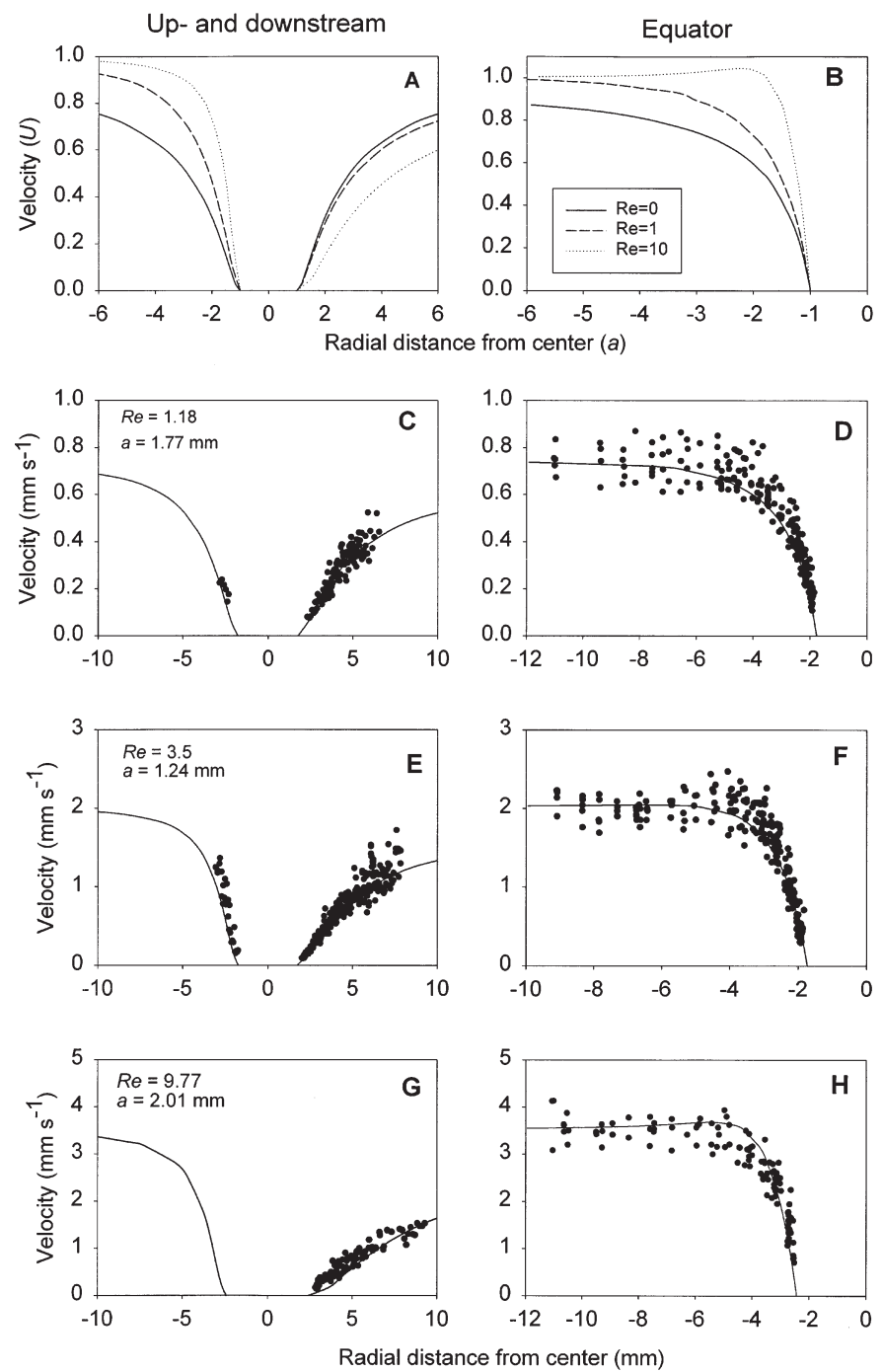

Fig. 2. Observed and computed velocity profiles up- and downstream of the sphere and off the sphere's equator at various Re. Lines refer to velocities computed from the model, circles to observed velocities. In (A) and (B) distances and computed velocities are in units of sphere radii $(a)$ and sinking velocity $(U)$ 


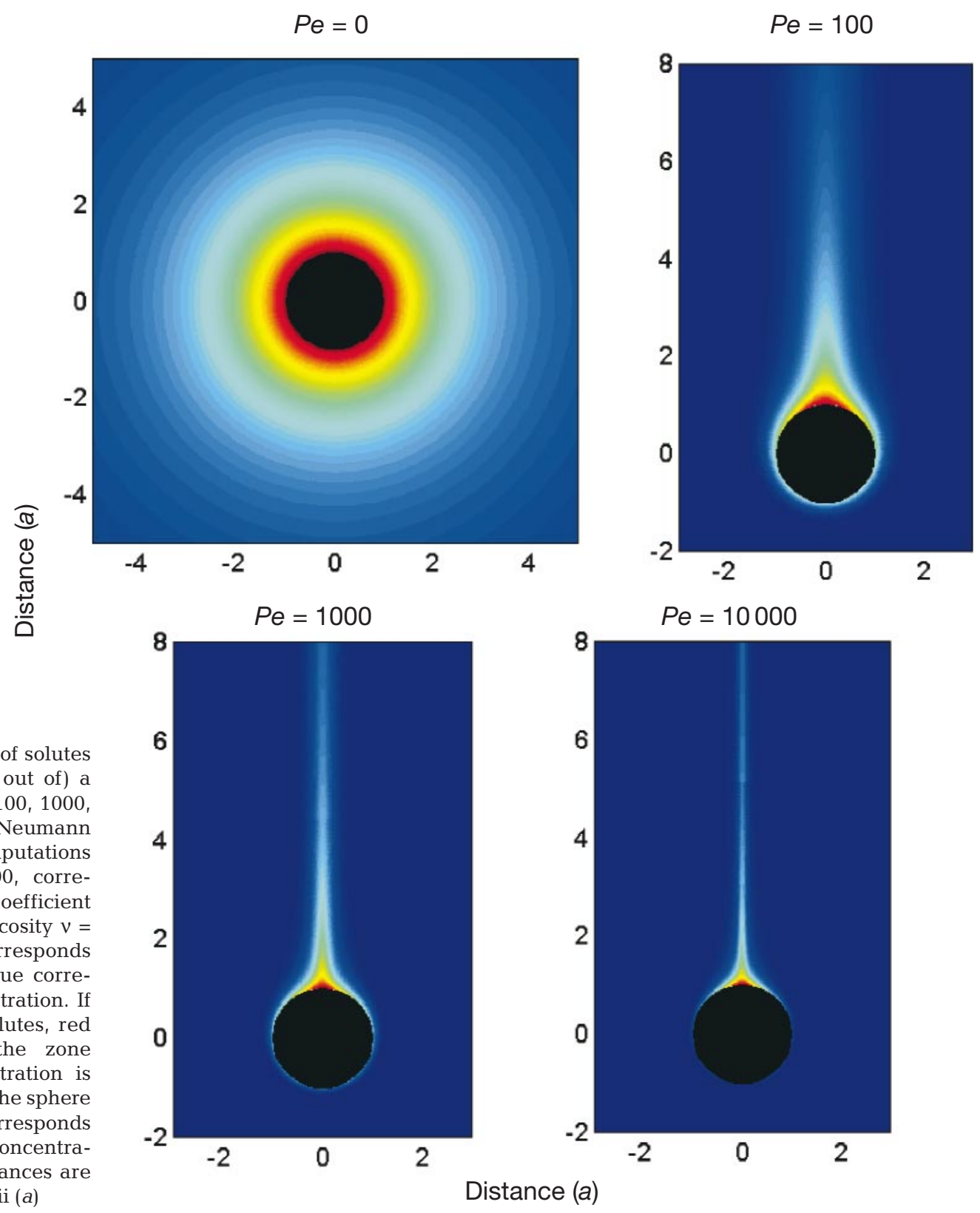

predicted from the model, as does the inner parts of the downstream concentration gradients (Fig. 4, here shown for a constant flux per surface area over the entire aggregate). However, further downstream, the observed oxygen concentration approaches ambient concentration faster than predicted and the tail thus appears much shorter than predicted by the model (Fig. 4). We ascribe this to the flow instabilities mentioned earlier: a slight deviation of the flow from perfectly vertical moves the centre of the slender plume to the side of the electrode. Thus, as for the flow field, the concentration field is very similar to that predicted, particularly in the vicinity of the sphere. The experi- mental set-up used here and in several previous experiments (Ploug et al. 1997, 1999a,b, Ploug \& Jørgensen 1999) is, hence, representative of the flow and diffusion field in the vicinity of a naturally sinking aggregate. There was also no evidence for artefacts in the measured oxygen concentrations induced by the microsensor in the diffusive boundary layer around a sphere as reported in sediment studies (Glud et al. 1994). This is likely explained by the fact that the electrode, which is much smaller than the aggregate itself, is directed parallel to the flow, and that the 3-dimensional flow and diffusion field around aggregates are apparently not sensitive to the electrode. 

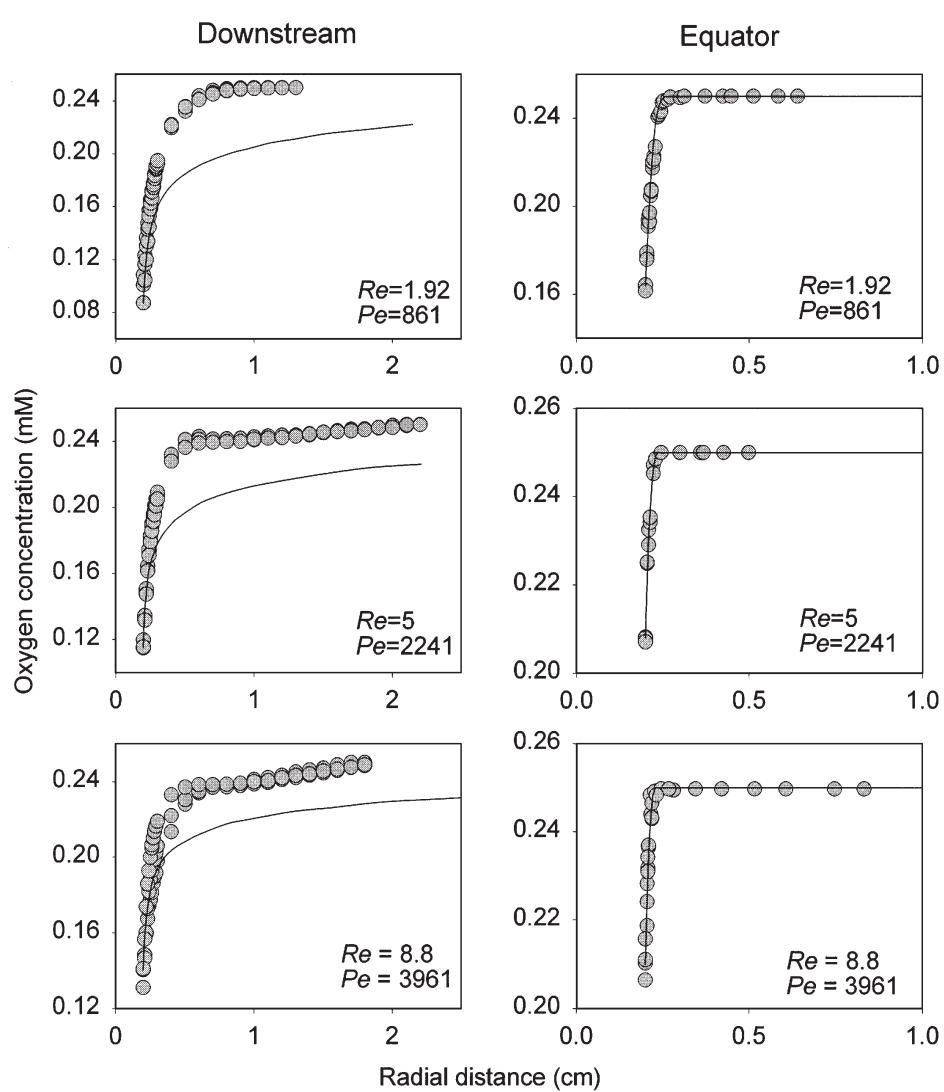

Fig. 4. Examples of modelled (lines) and observed (circles) concentrations of oxygen downstream and off the equator of yeast aggregates suspended in a laminar flow. Computed values are for a Neumann boundary condition

\section{Mass transfer}

The dimensionless Sherwood number $(S h)$ is the ratio of total mass transport (diffusive + advective) to that for pure diffusion to/from the sphere. It thus describes the relative enhancement of solute fluxes due to advection. It is defined only for a transportlimited system (i.e., a Dirichlet boundary condition). For pure diffusion, $S h=1$. The $S h$ depends on both the $P e$ and the Re. The Sh has previously been used to examine the effect of advection (swimming, sinking) on solute uptake in unicellular organisms (e.g., Kiørboe 1993, Karp-Boss et al. 1996 and references therein) and in sinking particles, aggregates or colonial organisms (Logan \& Alldredge 1989, Jannasch et al. 1996, Ploug et al. 1999b). It is consequently a useful property in biological oceanography.

In considering the movement of solutes leaking out of or consumed by sinking aggregates, we computed $S h$ for different values of $P e$ and Re. Our model accurately replicates previous attempts to examine the dependency of $\mathrm{Sh}$ on $\mathrm{Pe}$ when a Stokes' flow is assumed
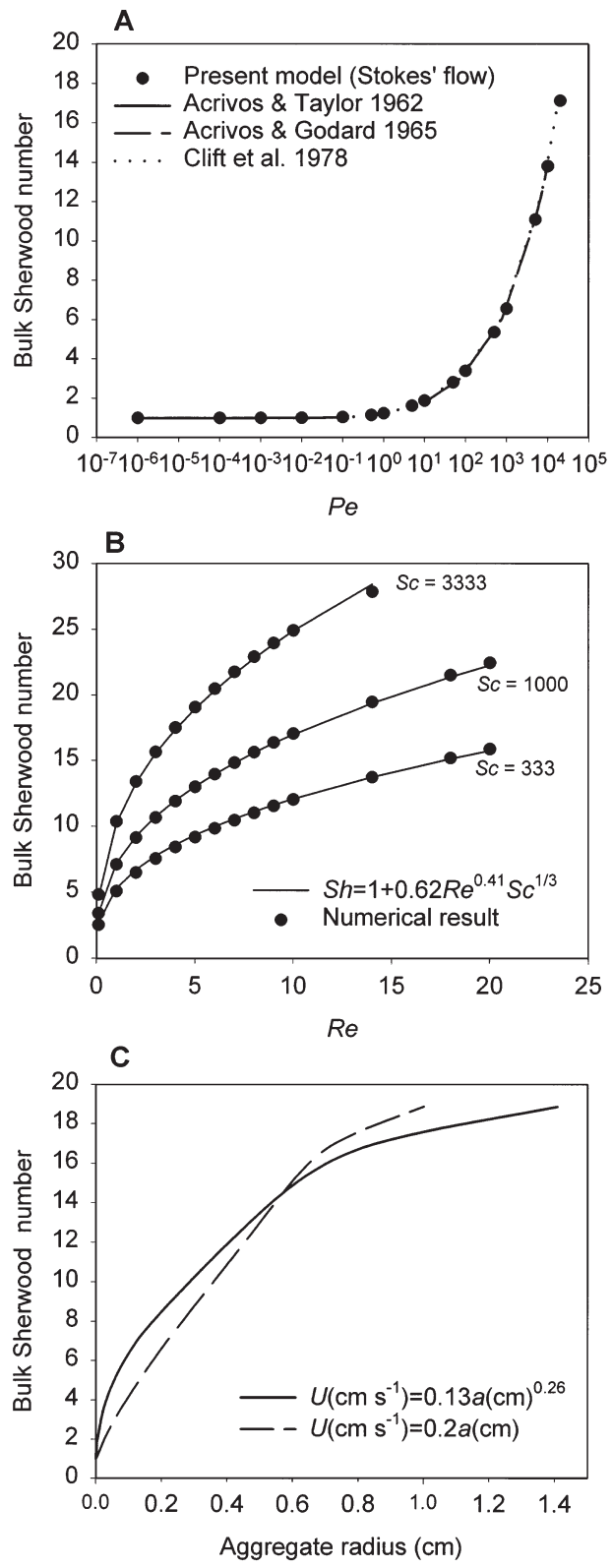

Fig. 5. (A) Computed bulk $S h$ as a function of $P e$ compared with previous numerical and analytical results, where Stokes' flow has been assumed. Computations with the present model were made for Stokes' flow. A Dirichelt boundary condition has been employed in both the present computations as well as in those from the literature. The previously reported results are the asymptotic analytical solution for $P e \ll 1$ : $S h=$ $1+{ }^{1} / 2 P e+1 / 2 P e^{2} \ln (P e)($ Acrivos \& Tayler 1962); for $P e \gg 1$ : $S h=0.6245 P e^{1 / 3}+0.461$ (Acrivos \& Goddard 1965) $;$ and the numerically determined approximation (all Pe): $S h=1 / 2[1+$ $(1+2 P e)^{1 / 3}$ ] (Clift et al. 1978) (see Karp-Boss et al. 1996). (B) Computed bulk $S h$ versus $R e$ for various $S c$. Circles are numerical results, solid lines are the fitted relation. (C) Computed bulk $S h$ as a function of aggregate size employing 2 different sinking velocity versus size relations (Eqs. 4a,b). Computations were made for $S C=1000$, which would be typical for small biological molecules $\left(D \approx 10^{-5} \mathrm{~cm}^{2} \mathrm{~s}^{-1}\right)$ in seawater $\left(v \approx 10^{-2} \mathrm{~cm}^{2} \mathrm{~s}^{-1}\right)$. For $S C=300$, Sh is up to $30 \%$ less, and for $S C=4000$, Sh is up to $60 \%$ higher than shown here 
(i.e. very low $R e$ ) (Fig. 5A). In this ideal situation the Sh depends solely on Pe. However, the assumption of Stokes' flow becomes increasingly violated with increasing $R e$ (and $P e$ ). We therefore computed $S h$ for varying $R e$ (0.1 to 20 ) and for $S c$ between 300 and 4000 (Fig. 5B), which encompasses the biologically relevant range. We fitted a relation of the form $S h=$ $1+\mathrm{a} R e^{\mathrm{b}} S C^{1 / 3}$ to the computed values (cf. Clift et al. 1978). The fitted relation agrees to within $5 \%$ of the numerical solution for $0.1<R e<20$ and $30<P e<$ 50000 :

$$
S h=1+0.619 R e^{0.412} S C^{1 / 3}
$$

The effect of a more correct representation of the flow becomes significant ( $>10 \%$ deviation) for $R e>1$ at $S C=1000$.

It is of relevance to examine how $S h$ depends on the size of a marine snow aggregate. Alldredge \& Gotschalk (1988, 1989) made in situ observations of sinking velocities of aggregates of various sizes, and provided the following empirical relationships between sinking velocity $(U)$ and equivalent radius $(a)$ :

$U\left(\mathrm{~cm} \mathrm{~s}^{-1}\right)=0.13 a(\mathrm{~cm})^{0.26}$

(Alldredge \& Gotschalk 1988)

$U\left(\mathrm{~cm} \mathrm{~s}^{-1}\right)=0.2 a(\mathrm{~cm})$

(Alldredge \& Gotschalk 1989)

Employing these relations together with Eq. (4), we estimated the variation in $S h$ with aggregate size for $S C=1000$ (Fig. 5c). Estimated $S h$ for typical marine snow aggregate sizes $(0.1$ to $1 \mathrm{~cm})$ vary between ca 4 and 20. Thus, to the extent that mass transfer is transport limited, solute transport is facilitated by a factor of this magnitude in sinking over non-sinking marine snow aggregates. In comparison, the Sh for unicellular sinking or swimming organisms typically fall in the range of 1 to 2 (Karp-Boss et al. 1996). Hence, advection is significantly more important for mass transfer to/from sinking aggregates than it is for sinking or swimming unicellular organisms.

So far we have considered only bulk $S h$, i.e. the average over the entire surface of the sphere. However, local values vary several-fold (Fig. 6A). The local Sh is the normalised concentration gradient at the sphere's surface, and it describes the solute distribution in the immediate vicinity of the sphere. It depends on the local thickness of the effective diffusive boundary layer $\left(\mathrm{DBL}_{\theta}\right)$

$$
\mathrm{DBL}_{\theta}=\frac{C_{\infty}-C_{r=a, \theta}}{\left.\frac{\partial C}{\partial r}\right|_{r=a, \theta}}
$$

and is defined as (Sherwood et al. 1975)

$$
S h_{\text {local }, \theta}=\frac{\left.a \frac{\partial C}{\partial r}\right|_{r=a, \theta}}{C_{\infty}-C_{r=a, \theta}}
$$

where $C_{r=a, \theta}$ and $C_{\infty}$ are the concentrations at the surface (in direction $\theta$ ) and far from the sphere, and $r$ is the radial distance from the centre of the sphere. In contrast to the bulk $S h$, local $S h$ is defined for both a
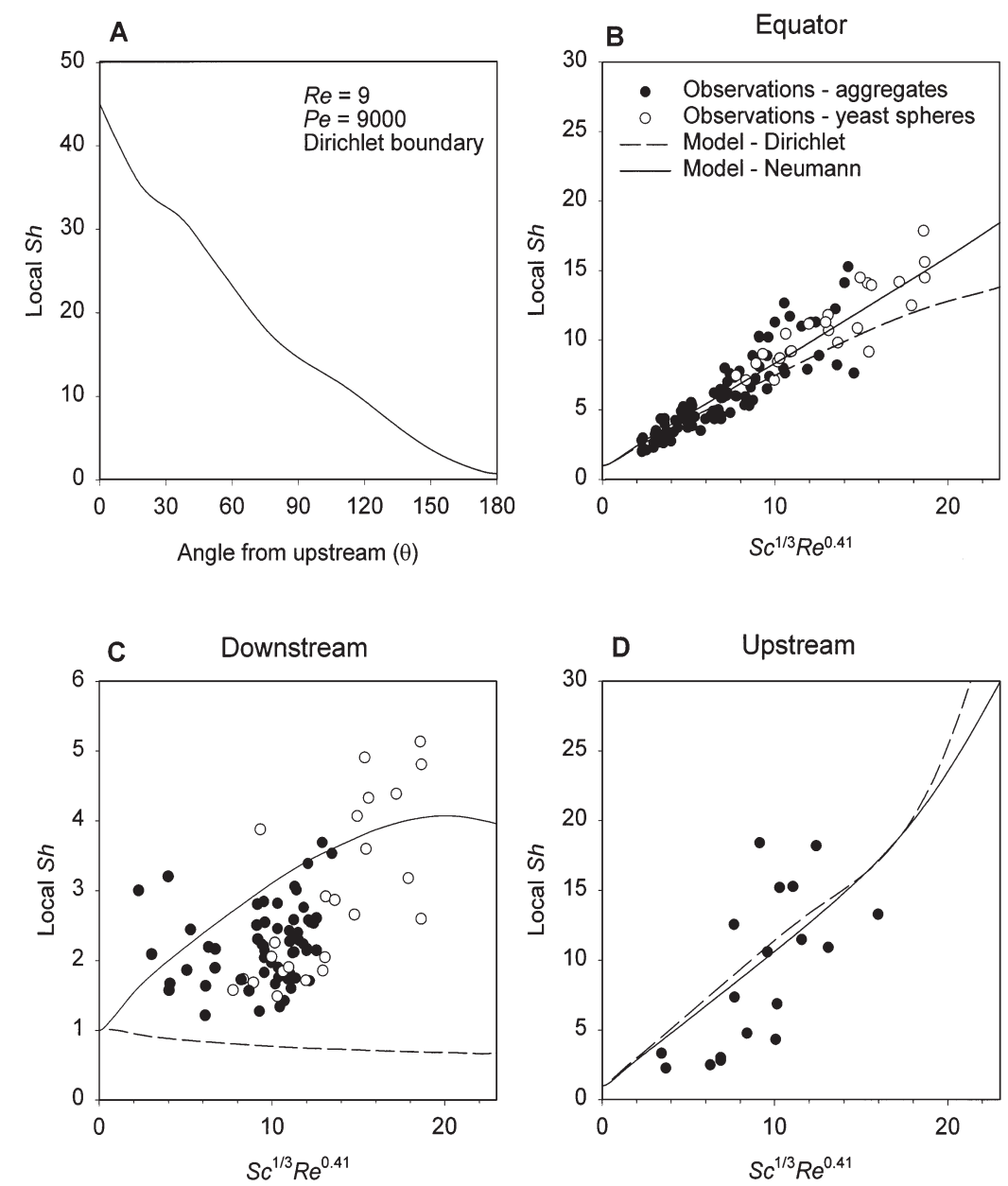

Fig. 6. (A) Example of how the local Sherwood number varies as a function of the position at the surface of the aggregate measured as angle from upstream $(\theta)$. The computation was made for a Dirichlet boundary condition at $R e=9$ and $P e=9000$. (B-D) Computed and observed local Sh upstream, downstream and off the equator of the aggregate as a function of $\mathrm{Pe}$ and Re. Computations (lines) were made with both a Dirichlet and a Neumann boundary condition. Open circles are for yeast spheres; solid circles are for lab-made and fieldcollected detritus aggregates 
reaction and a transport-limited system. For the Neumann boundary condition, the radial flux is constant over the surface, and variations in local Sh therefore reflect variations in surface concentration depending on the local DBL thickness. Similarly, variation in local Sh for a Dirichlet boundary condition reflects variation in radial fluxes depending on the local DBL thickness. Thus, during transport limitation, the flux of solutes is highest in the upstream direction where the local $S h$ is highest (Fig. 6A).

Observed local Sh numbers at the equator and upstream our near-spherical yeast aggregates agree well with those predicted, independent of whether we employ a Dirichlet or a Neumann boundary condition. (Fig. 6B,D). However, downstream from the aggregate the observed local $S h$ scatters between the local $S h$ predicted by the 2 alternative boundary conditions (Fig. 6C). This applies also to the lab-made and fieldcollected aggregates. Thus, neither boundary condition accurately applies to our aggregates, but they rather describe extremes enveloping the observations. Deviations from a Neumann boundary imply that the solute flux is transport limited and deviations from a Dirichlet boundary imply that it is reaction limited. The intermediate local Sh suggests that the oxygen uptake rate is both transport and reaction limited.

Even the local Sh measured in more irregular lab-made and field-sampled aggregates are well described by the model, and our observations are thus consistent with predictions for impermeable spheres. The apparent porosity of marine aggregates is high, greater than 0.9990 in $>5 \mathrm{~mm}$ diameter aggregates (Alldredge \& Gotschalk 1988). Flow through porous aggregates has so far not been verified experimentally and the impact of any potential flow has only been quantified from model calculations, which suggest that flow within large $(>7 \mathrm{~mm})$, porous and fast-sinking aggregates may increase mass transfer to attached cells within aggregates 1.0- to 2.1-fold compared to that to free-living cells (Logan \& Hunt 1987, Logan \& Alldredge 1989). The oxygen gradients around our $<6 \mathrm{~mm}$ large field-sampled marine aggregates, however, are well described by the model for impermeable aggregates to which mass transfer is potentially 4 - to 20 -fold enhanced during sedimentation. High abundance of transparent exopolymeric particles (TEP) within aggregates may have limited flow within these aggregates. However, recent studies of highly porous field-sampled aggregates have shown that flow through such aggregates may imply concentration gradients significantly different from those predicted by the model and a more efficient mass transfer (Ploug \& Hietanen unpubl.). Hence, mass transfer to sinking aggregates predicted and measured in the present study is conservative.

\section{Measuring biological rates in aggregates}

There are several implications of the model and our observations for estimating biological rates in aggregates. First, whether the flux or the concentration can be considered constant over the surface of the sphere (Neumann vs Dirichlet boundary condition) has implications for the calculation of solute fluxes in microsensor studies. Since our observations suggest that the flux may not be constant over the entire surface area, measurements should in principle be done at multiple locations of the aggregate surface and then be integrated to yield total solute flux (here equal to respiration rate). Unfortunately, this is very laborious. However, bulk $S h$ are very close to $S h$ measured along the equator, implying that measurements at this location can be considered representative.

The flow environment during incubation of aggregates may be important for the correct measurements of biological rates. It follows from the diffusionadvection equation (Eq. A7, Appendix 1) and the definition of the local Sh (Eq. 7), that the local steadystate surface concentration of solutes $\left(C_{r=a, \theta}\right)$ and/or the local flux per surface area $\left(J_{\theta}\right)$ varies with the local $S h$ :

$$
C_{\infty}-C_{r=a, \theta}=\frac{J_{\theta} \mathrm{a}}{D S h_{\mathrm{local}, \theta}}
$$

The exchange rate of solutes between an aggregate and its environment is limited either by the capacity of the organisms in the aggregate to consume or produce solutes (i.e., a Neumann boundary condition - the flux is constant and $C_{r=a, \theta}$ varies with the local $S h$ ) or by the transport capacity (i.e., a Dirichlet boundary condition $-C_{r=a, \theta}$ is constant and $J_{\theta}$ varies with the local $\left.S h\right)$.

If the aggregate's maximal uptake capacity of a solute exceeds the transport potential, then the flux becomes transport limited. It follows from Eq. (8) that the practical implication of this is that one may underestimate biological rates if advective transport is prevented and measurements are done on non-sinking aggregates in stagnant water. The magnitude of the $S h$ indicates the magnitude of the error. This may, for example, apply to measurements of aggregate respiration rates in sediment traps (see Banse 1990) or during experimental incubations (e.g., Simon et al. 1990). Observations on lab-made and on field-collected aggregates suggest that the biological rate may at times be partly transport limited (Fig. 6), even when the aggregate is suspended in a flow. Thus, a correct flow environment is a prerequisite for representative measurements.

If instead the solute flux is reaction limited, it follows from Eq. (8) that the solute concentration at the surface and, therefore, in the interior of the aggregate varies with the flow. Thus, concentrations of solutes that are 
produced or consumed (e.g., dissolved organic matter, inorganic nutrients, oxygen) by microorganisms in aggregates may be radically different between sinking and non-sinking aggregates. The magnitude of the local $S h$ indicates the size of the difference. A correct flow environment thus has consequences for the correct estimation of rates (e.g., bacterial growth rate, phytoplankton production) that depend on these concentrations. The use of inappropriate flow environments in the past may have led to the suggestion that the interior of aggregates can be anoxic (e.g., Paerl \& Bebout 1988) and to underestimates of bacterial growth rates in aggregates (see Ploug \& Grossart 2000). It has recently been shown that the oxygen concentration inside aggregates can be 9-fold lower when aggregates are resting on a solid surface compared to when they are sinking through the water column (Ploug \& Jørgensen 1999). Similarly, bacterial production measured on aggregates is 5- to 10 -fold higher when the aggregates are incubated individually and suspended as in the natural environment as compared to aggregates that are not maintained in suspension during incubation (Ploug \& Grossart 1999, Ploug 2001).

\section{Heterogeneity in the ocean}

Marine snow aggregates are both sinks and sources of solutes and they may thus influence the ambient distribution of solutes in the ocean. It has previously been pointed out that solutes are not evenly distributed in seawater, but that plumes of elevated concentrations of nutrients or dissolved organics may originate from zooplankton excretion events (Jackson 1980, Lehman \& Scavia 1982), from leakage from sinking diatoms (Jackson 1987, 1989), or from lysing unicellular organisms (Blackburn et al. 1998). Generally, small plumes from single cells do not persist very long because diffusion at the $\mu \mathrm{m}-\mathrm{mm}$ scale rapidly ( $\mathrm{s}-\mathrm{min}$ ) dissipates the locally elevated concentrations. Yet it has been demonstrated that chemotactic motile bacteria may take advantage of such plumes (Blackburn et al. 1998, Jackson 1989). Also, the significant enrichment of heterotrophic organisms on aggregates compared to their ambient concentrations may partly be explained by colonization through chemotactic localization of high concentration of organic matter by the organisms (Jackson 1989).

Marine snow aggregates may be important sources of dissolved organic matter because attached bacteria seem to solubilize particulate organic matter faster than they assimilate it (Smith et al. 1992, 1995, Grossart \& Simon 1998, Vetter et al. 1998). Hence, leaking and sinking aggregates generate trails of elevated concentrations of dissolved organic matter on a $\mathrm{cm}-\mathrm{m}$ scale during sedimentation (cf. Fig. 3). Because the diffusion time increases with distance squared, the larger spatial extension (width) of snow-generated solute plumes makes these more 'diffusion resistant' than the small plume generated by a lysing protist, for example, and may thus have a disproportionate influence on ambient solute distributions and on osmotrophs.

To illustrate the ambient effect of a leaking aggregate we may estimate the volume of the plume with amino acid concentrations high enough that bacterial growth might be affected (Fig. 7). Based on the sizespecific organic nitrogen content of aggregates (Alldredge 1998) and a PON-specific release of amino acids of $0.20 \mathrm{~d}^{-1}$ (Smith et al. 1992), the leakage rate of dissolved amino acids from a $0.5 \mathrm{~cm}$ aggregate sinking at $0.1 \mathrm{~cm} \mathrm{~s}^{-1}$ (cf. Eq. 5a,b) can be estimated to be about $3.5 \times 10^{-13} \mathrm{~mol} \mathrm{~s}^{-1}$. Midwater bacterial populations respond by increased respiration and incorporation rates

Fig. 7. 3-dimensional representation of the plume of elevated amino acid concentration in the wake of a sinking aggregate. In this example, a $0.5 \mathrm{~cm}$ radius particle sinking at $0.1 \mathrm{~cm} \mathrm{~s}^{-1}$ and leaking amino acids at $3.5 \times 10^{-13} \mathrm{~mol} \mathrm{~s}^{-1}$ has been described. The depicted plume corresponds to concentrations elevated by $3 \times$ $10^{-8} \mathrm{M}$ above ambient concentration. All distances are in units of sphere radii $(a)$

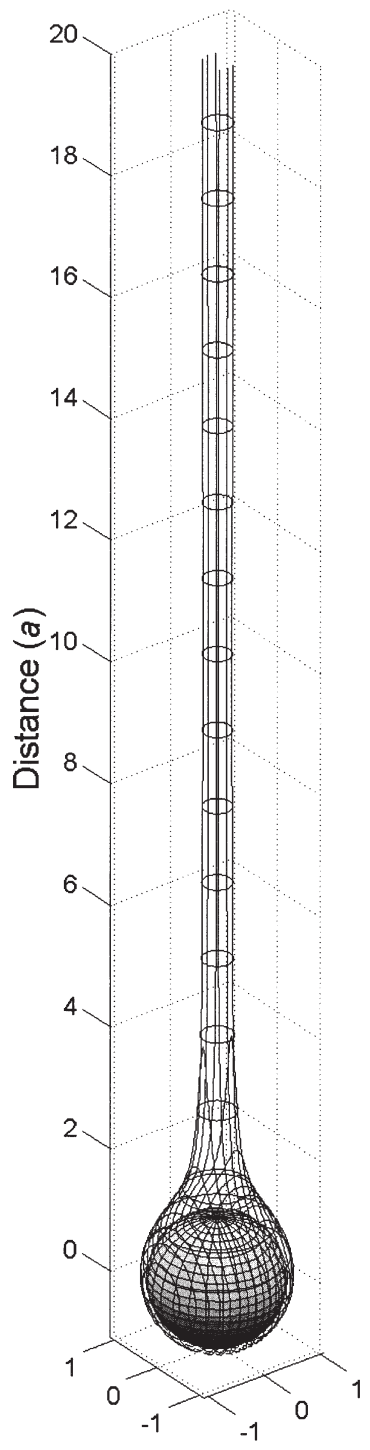


at amino acid additions of $2 \times 10^{-9} \mathrm{~mol} \mathrm{l}^{-1}$, neritic surface populations at $>1.5 \times 10^{-8} \mathrm{~mol} \mathrm{l}^{-1}$ (Carlucci et al. 1986) and oligotrophic surface populations at $\sim 10^{-9} \mathrm{~mol}$ $\mathrm{l}^{-1}$ (Suttle et al. 1991). Atema (1988) reported a halfsaturation constant for amino acid uptake of about $3 \times$ $10^{-8} \mathrm{M}$ in marine bacteria, and Suttle et al. (1991) found half-saturation constants an order of magnitude less. From the model we can calculate that the volume of the plume with concentrations exceeding a half saturation concentration of $3 \times 10^{-8} \mathrm{M}$ is about 100 times the volume of the aggregate itself (Dirichlet boundary condition). This effect on solute distribution may thus have implications for bacterial growth rates in a much larger volume of water than that occupied by the aggregate itself. The estimate is very sensitive to the leakage rate of amino acids, and the affected volume will shrink considerably with decreasing leakage rate, e.g., to $10 \times$ aggregate volume if the leakage rate is $0.1 \mathrm{~d}^{-1}$. However, the estimate is relatively insensitive to the size of the aggregate within the typical size range of aggregates ( $\sim 0.1$ to $1.0 \mathrm{~cm}$ radius). Volume concentrations of aggregates vary widely. The numbers compiled by Alldrege \& Silver (1988) for the upper ocean range between 10 and $7000 \mathrm{ppm}$ with a geometric mean of ca $300 \mathrm{ppm}(=0.03 \%$ of the water column occupied by aggregates). If a water volume $100 \times$ the volume of the aggregates holds amino acid concentrations high enough to affect bacterial growth, then leakage from sinking aggregates may create significant microniches for free-living bacteria. However, leakage would only in rare occasions (with extreme aggregate abundances) influence bulk water concentrations of solutes. The characteristic time scale of the plume is defined as the ratio of plume length to aggregate sinking velocity. The length of the considered plume for our $0.5 \mathrm{~cm}$ aggregate is $80 \mathrm{~cm}$; hence the time scale is $13 \mathrm{~min}$. Due to the reduced flow of water in the vicinity of the sinking aggregated (cf. Figs. $1 \& 2$ ), the residence time of bateria within the plume may be much longer. Chemosensory motile bacteria may further increase their residence time, as has been demonstrated for bacteria in plumes associated with sinking phytoplankton cells (Jackson 1989), and may, in combination with the reduced flow, make the plumes much more important to bacteria than the plume volume would indicate. Similarly, remineralization products leaking out of aggregates may have an effect on phytoplankton growth in the euphotic zone. Judged from reported concentrations of silicic acid and ammonium inside aggregates - orders of magnitude higher than ambient concentrations (Shanks \& Trent 1979, Brzezinski \& Alldredge 1997) — this effect may well be significant. Another potential implication of the long slender chemical trail at the rear of the sinking aggregate is that it may help zooplankters to remotely locate, colonize and feed on marine snow particles (Kiørboe \& Thygesen 2001, in this issue), thus further contributing to the remineralization of aggregated organic particles.

One could imagine that turbulence would dissipate such plumes rapidly. However, direct observations in the ocean of colour trails left by small sinking particles leaking fluorescein demonstrate that such trails may persist for many minutes and be meters long in calm water and in the pycnocline, although they may bend and become filamentous due to shear and turbulence (Woods 1968, 1971). The full implications of the heterogeneity in solute distributions due to leaking and sinking particles to microorganism population dynamics, aggregate remineralization, ocean carbon turnover, and the effects of turbulence on these processes have yet to be examined. The model developed here may help us do so.

Acknowledgements. We appreciate the critical comments of George Jackson on the manuscript and his help in generating Fig. 7. The work was supported by grants from the Danish Natural Science Research Council (9801391) and from the Carlsberg Foundation (J.970213/20-886 and J.980511/ 20-513).

Appendix 1. Numerical solution of the Navier-Stokes' and diffusion-advection equations

We consider a spherical co-ordinate system which follows the sphere, where $r$ denotes the distance from the centre of the sphere, $\phi$ denotes longitude, and $\theta$ latitude with $\theta=0$ pointing downstream.

Model of the fluid flow. The fluid flow around the sphere is assumed to be incompressible, axially symmetric and in steady state and can therefore be described by the timeindependent stream function denoted $\psi(r, \theta)$ (Acheson 1990). The physical significance of the stream function is that $2 \pi \psi(r, \theta)$ is the flow through the circle in 3-dimensional space given by $r$ and $\theta$. With these assumptions, the NavierStokes' equations can be formulated as a fourth order non- linear partial differential equation in the stream function (van Dyke 1964):

$$
\left\{L^{2}-\frac{R e \cdot a}{r^{2} \sin \theta}\left[\frac{\partial \psi}{\partial \theta} \frac{\partial}{\partial r}-\frac{\partial \psi}{\partial r} \frac{\partial}{\partial \theta}+\left(2 \cot \theta \frac{\partial \psi}{\partial r}-\frac{2}{r} \frac{\partial \psi}{\partial \theta}\right)\right]\right\} \zeta=0
$$

where $a$ is the radius of the sphere, $\quad \zeta=L^{2} \psi$ and the operator $L^{2}$ is shorthand for

$$
L^{2}=\frac{\partial^{2}}{\partial r^{2}}+\frac{1}{r^{2}} \frac{\partial^{2}}{\partial \theta^{2}}-\frac{\cot \theta}{r^{2}} \frac{\partial}{\partial \theta}
$$

The literature typically uses the notation $D^{2}$ for our $L^{2}$ and states these equations in dimensionless co-ordinates. The 
boundary conditions associated with this equation expresses that the flow velocity is zero at the surface of the sphere, and approach free flow far from the sphere:

$$
\begin{array}{ll}
\psi=0 & \text { at the surface of the sphere } \\
\frac{\partial \psi}{\partial r}=0 & \text { at the surface of the sphere } \\
D^{2} \psi \rightarrow 0 & \text { as } r \rightarrow \infty, \text { and } \\
\psi \rightarrow \frac{1}{2} U r^{2} \sin ^{2} \theta & \text { as } r \rightarrow \infty
\end{array}
$$

Given a stream function, the associated flow field is found by differentiating:

$$
u_{\phi}=0, \quad u_{r}=\frac{1}{r^{2} \sin \theta} \frac{\partial \psi}{\partial \theta}, \quad u_{\theta}=-\frac{1}{r \sin \theta} \frac{\partial \psi}{\partial r}
$$

Here $u_{\phi}, u_{\theta}$ and $u_{r}$ are the components of the flow in the latitudinal, longitudinal and radial direction, respectively. Finally, the vorticity, $\nabla \times u$, was computed as another descriptor of the flow field.

The advection-diffusion equation. At a point in the fluid, the flux of the solute is

$$
u \cdot C-D \nabla C
$$

Here $D$ is the diffusivity and $C$ is the concentration of the solute while $u$ is the flow vector. The first term is advection and the second term is diffusion. Conservation of the solute implies that this flux is divergence free, i.e. the concentration satisfies the advection-diffusion equation

$$
u \cdot \nabla C-D \nabla^{2} C=0
$$

Here we have used that the flow is divergence free, i.e. $\nabla \cdot u=0$

Eq. (A7) was solved with the following boundary conditions: Far from the sphere, the concentration approaches the ambient concentration $C_{\infty}$ :

$$
\left.C\right|_{r=\infty}=C_{\infty}
$$

We investigated 2 alternative boundary conditions, i.e., the Dirichlet condition, where the concentration is fixed and constant over the surface of the sphere:

$$
\left.C\right|_{r=a}=C_{0}
$$

and the Neumann condition, where the radial derivative (hence the flux) is fixed and constant over the surface of the sphere:

$$
\left.\frac{\partial C}{\partial r}\right|_{r=a}=C_{0}
$$

Discretisation. The advection-diffusion equation was discretised using a finite difference scheme on a rectangular grid in polar co-ordinates $(r, \theta)$.

Using circular symmetry, we are interested in the solution in the region

$$
\theta \in[0, \pi], \quad r \in\left[a, R_{\max }\right], \quad \phi=0
$$

Here we should in principle take $R_{\max }=\infty$ but we will reconcile with $R_{\max }$ large.
The computational grids are rectangular in the spherical coordinates $r, \theta$, but are not uniform. In the radial direction, the spacing is logarithmic, i.e.

$$
r_{i}=a \cdot h^{i-1}, \quad i=1, \ldots, M
$$

In the latitudinal direction, the spacing is given by

$$
\theta_{j}+\gamma \sin \left(\theta_{j}\right)=k j-\frac{k}{2}, \quad j=1, \ldots, N
$$

Here the step sizes $h$ and $k$ are determined by $a \cdot h^{M-1}$ and $k N=\pi$. The parameter $\gamma \in[0,1]$ makes the grid denser downstream than upstream, which is useful for high Peclet numbers.

The partial differential equations were discretised on these computational grids using finite difference schemes. The Navier-Stokes equations for the flow use a fourth-order central difference scheme on a grid with $\gamma=0$, i.e. the angle $\theta$ is equidistantly spaced. The advection-diffusion equation for the concentration uses a third/fourth order upwind schemes with $\gamma=0.7$. This grid has a density downstream which is $(1+0.7) /(1-0.7) \approx 5.7$ times higher than upstream. The use of upwind schemes is necessary to avoid unphysical unboundedness (ripples) in the solution; the high order reduces numerical diffusion. Since we use different grids for the flow and the concentrations, the flow field is interpolated onto the diffusion grid before solving the advection-diffusion equation. This is done using cubic splines.

The boundary conditions applied at $r=R_{\max }$ are for the flow equations that the stream function equals that corresponding to free flow, and that the vorticity is zero. For the advection-diffusion equation we set $C=C_{\infty}$ at $R=R_{\max }$. Experimenting with different values of $R_{\max }$ showed that this approximation is not critical, in particular not for high Peclet numbers. The results presented here are computed for $R_{\max } \geq 100$, which for all practical purposes makes the results independent of $R_{\max }$.

Solution of the algebraic equation systems. The discretisation transforms the partial differential equations into algebraic equations. The advection-diffusion equation is linear and was solved in 1 step using a solver for sparse matrix systems, which is built into Matlab. The Navier-Stokes' equations are non-linear and were solved iteratively by constructing a sequence $\psi_{i}$ and $\zeta_{i}$ as the solutions to Dirichlet problems. The iteration law is as follows: First, given $\zeta_{i}$ we find $\psi_{i}$ by solving the partial differential Eq. (A2) with the boundary conditions, Eqs. (A3) \& (A6). Then we find $\zeta_{i+1}$ by solving Eq. (A1) with boundary condition Eq. (A5) at the exterior boundary. The inner boundary condition on $\zeta_{i+1}$ is not specified by the physics but must correspond to Eq. (A4). We therefore guess a function $\xi_{i+1}$ on the inner boundary and use the condition $\zeta_{i+1}=\xi_{i+1}$ at the inner boundary. In the next iteration we evaluate the guess $\xi_{i+1}$ by observing how well $\psi_{i+1}$ satisfies the boundary condition in Eq. (A4), and use a technique originating from stochastic adaptive control to adjust the boundary condition $\zeta_{i+1}$. The iteration is stopped when the boundary condition Eq. (A4) is satisfied up to a specified tolerance and $\psi_{i}$ changes less than a specified tolerance from one iteration to the next. 


\section{LITERATURE CITED}

Acheson DJ (1990) Elementary fluid dynamics. Clarendon Press, Oxford

Acrivos A, Goddard JD (1965) Asymptotic expansions for laminar forced-convection heat and mass transfer. J Fluid Mech 23:273-291

Acrivos A, Taylor TD (1962) Heat and mass transfer from single spheres in Stokes flow. Physics Fluid 5:387-394

Alldredge AL (1976) Discarded appendicularian houses as asources of food, surface habitats, and particulate organic matter in planktonic environments. Limnol Oceanogr 21: $14-23$

Alldredge AL (1998) The carbon, nitrogen and mass content of marine snow as a function of aggregate size. Deep-Sea Res 45:529-541

Alldredge AL, Gotschalk C (1988) In situ settling behaviour of marine snow. Limnol Oceanogr 33:339-351

Alldredge AL, Gotschalk C (1989) Direct observations of the mass flocculation of diatom blooms: characteristics, settling velocity and formation of diatom aggregates. DeepSea Res 36:159-171

Alldredge AL, Silver MW (1988) Characteristics, dynamics and significance of marine snow. Prog Oceanogr 20:42-82

Atema J (1988) Distribution of chemical stimuli. In: Atema J, Fay RR, Popper AN, Tavolga WN (eds) Sensory biology of aquatic animals. Springer-Verlag, New York, p 29-56

Banse K (1990) New views on the degradation and disposition of organic particles as collected by sediment traps in the open sea. Deep-Sea Res 37:1177-1195

Blackburn N, Fenchel T, Mitchell J (1998) Microscale nutrient patches in planktonic habitats shown by chemotactic bacteria. Science 282:2254-2256

Broecker WS, Peng TH (1974) Gas exchange rates between air and sea. Tellus 26:21-35

Brzezinski MA, Alldredge AL (1997) Silica cycling within marine snow. Limnol Oceanogr 42:1706-1713

Carlucci AF, Craven DB, Robertson KJ, Henrichs SM (1986) Microheterotrophic utilization of dissolved free amino acids in depth profiles of Southern California Borderland basin waters. Oceanol Acta 9:89-96

Caron DA, Davis PG, Laurance P, Sieburth JMcN (1982) Heterotrophic bacteria and bacterivorous protozoa in oceanic macroaggregates. Science 218:795-797

Chang EJ, Maxey MR (1994) Unsteady flow about a sphere at low to moderate Reynolds number. Part 1. Oscilatory motion. J Fluid Mech 277:347-379

Clift R, Grace JR, Weber ME (1978) Bubbles, drops and particles. Academic Press, New York

Cronenberg CHC (1994) Biochemical engineering on a microscale: biofilms investigated with needle-type glucose sensors. PhD thesis, University of Amsterdam

Csanady GT (1986) Mass transfer to and from small particles in the sea. Limnol Oceanogr 31:237-248

Dennis SCR, Walker JDA (1971) Calculation of the steady flow past a sphere at low and moderate Reynolds numbers. J Fluid Mech 48:771-789

Fowler SW, Knauer GA (1986) Role of large particles in the transport of elements and organic compounds through the ocean water column. Prog Oceanogr 16:147-194

Glud RN, Gundersen, JK, Revsbech NP, Jørgensen BB (1994) Effects on the benthic diffusive boundary layer imposed by microelectrodes. Limnol Oceanogr 39:462-467

Grossart HP, Simon M (1998) Bacterial colonization and microbial decomposition of limnetic organic aggregates (lake snow). Mar Ecol Prog Ser 15:127-140
Jackson GA (1980) Phytoplankton growth and zooplankton grazing in oligotrophic oceans. Nature 284:439-441

Jackson GA (1987) Simulating chemosensory response of marine microorganisms. Limnol Oceanogr 32:1253-1266

Jackson GA (1989) Simulation of bacterial attraction and adhesion to falling particles in an aquatic environment. Limnol Oceanogr 34:514-530

Jannasch HW, Honeyman BD, Murray JW (1996) Marine scavenging: the relative importance of mass transfer and reaction rates. Limnol Oceanogr 41:82-88

Johnson TA, Patel VC (1999) Flow past a sphere up to Reynolds number of 300. J Fluid Mech 378:19-70

Karp-Boss L, Boss E, Jumars PA (1996) Nutrient fluxes to planktonic osmotrophs in the presence of fluid motion. Oceanogr Mar Biol Annu Rev 34:71-107

Kiørboe T (1993) Turbulence, phytoplankton cell size, and the structure of pelagic food webs. Adv Mar Biol 29:1-72

Kiørboe T (2000) Colonization of marine snow aggregates by invertebrate zooplankton: abundance, scaling, and possible role. Limnol Oceanogr 45:479-484

Kiørboe T, Thygesen UH (2001) Fluid motion and solute distribution around sinking marine snow aggregates. II. Implications for remote detection by colonizing zooplankters. Mar Ecol Prog Ser 211:15-25

Kiørboe T, Titelman J (1998) Feeding, prey selection and encounter mechanisms in the heterotrophic dinoflagellate Noctiluca scintillans. J Plankton Res 20:1615-1636

Knauer G, Hebel D, Cipriano F (1982) Marine snow: major site of primary production in coastal waters. Nature 300: 630-631

Lehman JT, Scavia D (1982) Microscale nutrient patches produced by zooplankton. Proc Natl Acad Sci USA 79: 5001-5005

Logan BE, Alldredge AL (1989) Potential for increased nutrient uptake by flocculating diatoms. Mar Biol 101:443-450

Logan BE, Hunt JR (1987) Advantage to microbes of growth in permeable aggregates in marine systems. Limnol Oceanogr 32:1034-1048

Martin JH, Knauer GA, Karl DM, Broenkow WW (1987) VERTEX: carbon cycling in the northeast Pacific. Deep-Sea Res 38:267-285

Pace ML, Knauer GL, Karl DM, Martin JH (1987) Primary production, new production and vertical fluxes in the eastern Pacific Ocean. Nature 325:803-804

Paerl HW, Bebout BM (1988) Direct measurements of $\mathrm{O}_{2}$-depleted microzones in marine oscillatoria. Relation to $\mathrm{N}_{2}$-fixing capabilities. Science 241:441-445

Ploug H, Grossart HP (1999) Bacterial production and respiration on suspended aggregates - a matter of the incubation method. Aquat Microb Ecol 20:21-29

Ploug H, Grossart HP (2000) Bacterial growth and grazing on diatom aggregates: respiratory carbon turnover as a function of aggregate size and sinking velocity. Limnol Oceanogr 45:1467-1475

Ploug H, Jørgensen BB (1999) A net-jet flow system for mass transfer and microsensor studies in sinking aggregates. Mar Ecol Prog Ser 176:279-290

Ploug H, Kühl M, Buchholz B, Jørgensen BB (1997) Anoxic aggregates - an ephemeral phenomenon in the ocean. Aquat Microb Ecol 13:285-294

Ploug H, Grossart HP, Azam F, Jørgensen BB (1999a) Photosynthesis, respiration and carbon turn-over in sinking marine snow from surface waters of Southern California: implications for the carbon cycle in the ocean. Mar Ecol Prog Ser 179:1-11

Ploug H, Stolte W, Epping EHG, Jørgensen BB (1999b) Diffusive boundary layers, photosynthesis and respiration of 
colony-forming plankton alga, Phaeocystis sp. Limnol Oceanogr 44:1949-1958

Revsbach NP (1989) An oxygen microelectrode with a guard cathode. Limnol Oceanogr 34:474-478

Shanks AL, Trent JD (1979) Marine snow: microscale nutrient patches. Limnol Oceanogr 24:850-854

Shanks AL, Walters K (1997) Holoplankton, meroplankton, and meiofauna associated with marine snow. Mar Ecol Prog Ser 156:75-86

Sherwood TK, Pigford RL, Wilke CR (1975) Mass transfer. McGraw-Hill Book Company, New York

Simon M, Alldredge AL, Azam F (1990) Bacterial carbon dynamics on marine snow. Mar Ecol Prog Ser 65:205-211

Smith DC, Silver MW, Alldredge AL, Azam F (1992) Intensive hydrolytic activity on marine aggregates and implications for rapid particle dissolution. Nature 359:139-141

Smith DC, Steward GF, Long RA, Azam F (1995) Bacterial mediation of carbon fluxes during a diatom bloom in a mesocosm. Deep-Sea Res 42:75-97

Editorial responsibility: Otto Kinne (Editor),

Oldendorf/Luhe, Germany
Suttle CA, Chan AM, Furman JA (1991) Dissolved free amino acids in the Saragasso Sea: uptake and respiration rates, turnover times, and concentrations. Mar Ecol Prog Ser 70: 189-199

Turley CM, Mackie PJ (1994) Biogeochemical significance of attached and free-living bacteria and flux of particles in the NE Atlantic Ocean. Mar Ecol Prog Ser 115:191-203

van Dyke M (1964) Pertubation methods in fluid mechanics. Academic Press, London

Vetter YA, Deming JW, Jumars PA, Krieger-Brockett BB (1998) A predictive model of bacterial foraging by means of freely released extracellular enzymes. Microb Ecol 36: 75-92

Woods JD (1968) Wave-induced shear instability in the summer thermocline. J Fluid Mech 32:791-800

Woods JD (1971) Micro-oceanography. In: Woods JD, Lythgoe JN (eds) Underwater science. An introduction to experiments by divers. Oxford University Press, London, p 291-317

Submitted: March 20, 2000; Accepted: August 3, 2000

Proofs received from author(s): January 11, 2001 